\title{
ON THE NONSTANDARD REPRESENTATION OF MEASURES
}

\author{
BY \\ C. WARD HENSON
}

ABSTRACT. In this paper it is shown that every finitely additive probability measure $\mu$ on $S$ which assigns 0 to finite sets can be given a nonstandard representation using the counting measure for some * ${ }^{*}$-finite subset $F$ of ${ }^{*} S$. Moreover, if $\mu$ is countably additive, then $F$ can be chosen so that

$$
\int f d \mu=\operatorname{st}\left(\frac{1}{\|F\|} \sum_{p \in F} * f(p)\right)
$$

for every $\mu$-integrable function $f$. An application is given of such representations. Also, a simple nonstandard method for constructing invariant measures is presented.

Let $S$ be a set in some set theoretical structure $M$ and let ${ }^{*} S$ be the corresponding set in an enlargement ${ }^{*} \pi$ of $M$. Bernstein and Wattenberg have noted [2] that if $F$ is a ${ }^{*}$-finite subset of ${ }^{*} S$, then a finitely additive probability measure $\mu_{F}$ can be defined for all subsets $A$ of $S$ by

$$
\mu_{F}(A)=s t(\|* A \cap F\| /\|F\|) .
$$

They used this observation as the basis for a nonstandard proof of the theorem, due to Banach [1], which states that Lebesgue measure on $[0,1]$ can be extended to a totally defined (finitely additive) measure which is invariant under translations $(\bmod 1)$.

This paper concerns the representation of probability measures as nonstandard counting measures $\mu_{F}$. Let $\mu$ be any finitely additive probability measure which is defined on an algebra $B$ of subsets of $S$ and which satisfies $\mu(A)=0$ for each finite set $A$ in $B$. In $\$ 1$ it is shown that there exists a ${ }^{*}$-finite subset $F$ of ${ }^{*} S$ which satisfies $\mu=\mu_{F}$ on $B$. This has the consequence that for any bounded, $\mu$-integrable function $f$,

$$
\int f d \mu=s t\left(\frac{1}{\|F\|} \sum_{p \in F} * f(p)\right) .
$$

Received by the editors August 13, 1971.

AMS (MOS) subject classifications (1970). Primary 02H25, 26A98, 28A10; Secondary $28 \mathrm{~A} 70,46 \mathrm{~B} 10$. 
Moreover, if $\mathcal{B}$ is a $\sigma$-algebra and $\mu$ is countably additive, then $F$ can be chosen so that (2) holds for every $\mu$-integrable function.

Closely related to these results is a nonstandard representation for bounded linear functionals on the space $l_{\infty}$ of bounded sequences in $R$, which was given by Robinson [7]. In $\$ 2$ a straightforward extension of Robinson's result is used to give a nonstandard proof of a convergence result (Theorem 3 ) for bounded linear functionals on $C(X)$, where $X$ is a compact, Hausdorff space.

Also, in $\$ 3$ a nonstandard construction of invariant measures is given which yields a particularly simple proof of Banach's extension result for Lebesgue measure.

Preliminaries. The given structure $\pi$ is assumed to have the set $R$ of real numbers as an element (thus also the set $N$ of nonnegative integers). Moreover, the embedding $x \mapsto^{*} x$ of $M$ into ${ }^{*} \Pi$ is taken to be the identity on $R$. The standard part of a finite element $p$ of ${ }^{*} R$ is denoted by st $(p)$. If $p, q \in{ }^{*} R$, then $p={ }_{1} q$ means that $p-q$ is infinitesimal.

For each set $S$ in $M$ and each ${ }^{*}$-finite subset $F$ of ${ }^{*} S,\|F\|$ is the "cardinality" of $F$, in the sense of * $\pi$. That is, if $c$ is the function assigning to each finite subset $A$ of $S$ the cardinality of $A$, then $\|F\|={ }^{*} c(F)$. Alternately, $\|F\|$ is the smallest element $\omega$ of ${ }^{*} N$ for which there is an internal bijection between $F$ and $\left\{\omega^{\prime} \mid \omega^{\prime} \epsilon^{*} N\right.$ and $\left.\omega^{\prime}<\omega\right\}$. (For an introduction to the methods of nonstandard analysis see [5], [6] or [8].)

Given a set $S, \mathcal{P}(S)$ is the algebra of all subsets of $S$. Also, $l_{\infty}(S)$ is the linear space of all bounded, real valued functions on $S$, furnished with the sup norm. In this paper $\mu$ is a measure on $S$ if it is a nonnegative, finitely additive set function defined on an algebra of subsets of $S$. If $\mu$ is normalized to satisfy $\mu(S)=1$, then it is a probability measure. The notation $A \triangle B$ will be used for the symmetric difference, $(A \sim B) \cup(B \sim A)$, of two subsets of $S$.

1. Nonstandard representations. Let $\mu$ be a probability measure on $\mathscr{P}(S)$ and let $\phi$ be the linear functional on $l_{\infty}(S)$ defined by integration with respect to $\mu$. Then $\phi$ is a positive linear functional of norm 1 . Therefore, by the principal result of [7], there exist a ${ }^{*}$-finite subset $F$ of ${ }^{*} S$ and an internal function $\lambda$ from $F$ to ${ }^{*} R$ which satisfy

$$
s t\left(\sum_{p \in F}|\lambda(p)|\right)=1
$$

and, for each $f$ in $l_{\infty}(S)$,

$$
\phi(f)=s t\left(\sum_{p \in F} \lambda(p) * f(p)\right) .
$$


(Robinson's result [7] only covers the case $S=N$ explicitly, but his argument is easily extended to cover the general case.) Therefore the measure $\mu$ has the representation

$$
\mu(A)=\mathrm{st}\left(\sum_{p \in \epsilon^{*} A \cap F} \lambda(p)\right)
$$

Theorem 1 below states that, if $\mu(\{s\})=0$ for every $s \in S,(1)$ then $F$ can be chosen so that $\mu$ is represented as in (3), but with every $\lambda(p)$ equal to $1 /\|F\|$. That is, $\mu(A)=\mu_{F}(A)$ for every $A \subset S$.

Theorem 1. If $\mu$ is a probability measure on $\mathcal{P}(S)$ which satisfies $\mu(\{s\})=0$ for each $s \in S$, then there is $a^{*}$-finite set $F C^{*} S$ for which $\mu=\mu_{F}$.

Proof. Since ${ }^{*} \mathbb{K}$ is an enlargement of $\mathbb{M}$, there exists a ${ }^{*}$-finite subset $\mathbb{P}$ of $* \mathcal{P}(S)$ which satisfies ${ }^{*} A \in \mathbb{Q}$ for each $A \subset S$. For each internal subset $\mathcal{F}$ of $Q$, define

$$
E(\mathcal{F})=\bigcap\{E \mid E \in \mathcal{F}\} \cap \bigcap\left\{*^{*} \sim E \mid E \in \mathbb{Q} \sim \mathcal{F}\right\},
$$

so that the function taking $\mathcal{F}$ to $E(F)$ is internal. Let $\mathbb{Q}^{\prime}=\{E(\mathcal{F}) \mid \mathcal{F}$ is an internal subset of $\mathbb{P}\}$, so that $\mathbb{Q}^{\prime}$ is a ${ }^{*}$-finite set. Moreover, $\mathbb{Q}^{\prime}$ is a partition of ${ }^{*} S$, and each member of $\mathfrak{Q}$ is the union of an internal subset of $\mathbb{P}^{\prime}$.

Let $\omega=\left\|\mathbb{P}^{\prime}\right\|$ and choose $\tau \epsilon^{*} N$ so that $\omega^{2} / \tau$ is infinitesimal. For each $E$ in $\mathbb{Q}^{\prime}$ define $r(E)$ in ${ }^{*} N$ by the inequalities

$$
\tau(E) / \tau \leq{ }^{*} \mu(E)<(\tau(E)+1) / \tau .
$$

Then the function $E \mapsto \tau(E)$ on $\mathbb{Q}^{\prime}$ is internal. Moreover, if $E$ is a *finite element of $\mathbb{Q}^{\prime}$, then ${ }^{*} \mu(E)=0$, from which it follows that $\tau(E)=0$. Therefore there exists an internal function $f$ which is defined on $\mathcal{P}^{\prime}$ and which satisfies: For each $E$ in $\mathbb{Q}^{\prime}, f(E)$ is a ${ }^{*}$-finite subset of $E$ and $\|f(E)\|=\tau(E)$.

It will be shown that the set $F$ defined by

$$
F=\bigcup\left\{f(E) \mid E \in \mathbb{Q}^{\prime}\right\}
$$

satisfies the condition $\mu=\mu_{F}$. Since the elements of $\mathbb{Q}^{\prime}$ are pairwise disjoint, the elements of $\left\{f(E) \mid E \in \mathbb{Q}^{\prime}\right\}$ have the same property, and therefore,

$$
\|F\|=\sum_{E \in \mathbb{Q}^{\prime}} r(E) \text {. }
$$

Moreover, since the function ${ }^{*} \mu$ is ${ }^{*}$-finitely additive,

(1) The added condition on $\mu$ is only slightly more restrictive than necessary. Indeed, if $F$ is infinite and $s \in S$, then $\mu_{F}(\{s\}) \leq \operatorname{st}(1 /\|F\|)=0$. If $F$ is finite, say with $k$ elements, then $\mu_{F}$ is of the form $\mu=k-1\left(\mu_{1}+\ldots+\mu_{k}\right)$, where each of the measures $\mu_{j}$ takes on as values only 0 and 1 . 


$$
1=*_{\mu}(* S)=\sum_{E \in \mathbb{Q}^{\prime}} *_{\mu}(E) .
$$

Therefore, from the inequalities (4) follows

$$
\|F\| / \tau \leq 1<\|F\| / \tau+\omega / \tau,
$$

by summing over $E$. That is, by the choice of $\tau, \omega(\|F\| / \tau-1)$ is infinitesimal.

Now let $A$ be any element of $\mathfrak{Q}$ and let $\mathcal{F}$ be the collection of $E$ in $\mathbb{Q}^{\prime}$ which are subsets of $A$. Therefore $A$ is the union of $\mathcal{F}$, by the construction of $Q^{\prime}$. It follows that

$$
\|A \cap F\|=\sum_{E \in \mathcal{F}} \tau(E) \text {, and } *_{\mu}(A)=\sum_{E \in \mathcal{F}} *_{\mu}(E) \text {. }
$$

Therefore

$$
*_{\mu}(A)-\frac{\|A \cap F\|}{\|F\|}=\sum_{E \in \mathcal{F}}\left(*_{\mu}(E)-\frac{\tau(E)}{\|F\|}\right) .
$$

But for each $E$ in $Q^{\prime}$,

$$
\begin{aligned}
\mid *^{*}(E)- & \tau(E) /\|F\||\leq|{ }^{*} \mu(E)-\tau(E) / \tau|+| \tau(E) / \tau-\tau(E) /\|F\| \mid \\
& \leq 1 / \tau+(\tau(E) /\|F\|)|\|F\| / \tau-1| \leq 1 / \tau+|\|F\| / \tau-1| .
\end{aligned}
$$

Thus (5) implies

$$
\left|*_{\mu}(A)-\|A \cap F\| /\|F\|\right| \leq \omega / \tau+\omega|\|F\| / \tau-1|
$$

which is infinitesimal. In particular, for each $A \subset S$,

$$
\mu(A)={ }^{*} \mu(* A)=\operatorname{st}(\|* A \cap F\| /\|F\|)=\mu_{F}(A) .
$$

This completes the proof.

While Theorem 1, as stated, applies only to totally defined measures, it is valid for any probability measure $\mu$ which is defined on an algebra of subsets of $S$ and which assigns measure 0 to any finite set in its domain. This is because any such measure can be extended to a measure which satisfies the conditions of Theorem 1.

A different nonstandard representation for measures, based on partitions of * $S$ rather than ${ }^{*}$ finite subsets, has been developed and applied by Peter Loeb $[5],[6]$.

Lemma 1. Let $E$. be any *finite subset of ${ }^{*} S$ and let $F$ be an internal subset of $E$ which satisfies $\|F\| /\|E\|={ }_{1} 1$. Then $\mu_{F}=\mu_{E}$ on $\mathscr{P}(S)$ and

$$
\int f d \mu_{E}=\operatorname{st}\left(\frac{1}{\|F\|} \sum_{p \in F} * f(p)\right)
$$

for each $f$ in $l_{\infty}(S)$. 
Proof. Let $A$ be any subset of $S$. Then

$$
|\|* A \cap E\| /\|E\|-\|* A \cap F\| /\|E\|| \leq\|E \sim F\| /\|E\|={ }_{1} 0 .
$$

Therefore

$$
\mu_{E}(A)=\mathrm{st}\left(\|F\| /\|E\| \cdot\left\|^{*} A \cap F\right\| /\|F\|\right)=\mu_{F}(A)
$$

Now let $f$ be any element of $l_{\infty}(S)$, and define

$$
\psi(f)=s t\left(\frac{1}{\|F\|} \sum_{p \in F} * f(p)\right) .
$$

Then $\psi$ is a bounded linear functional on $l_{\infty}(S)$. Also, if $V$ is the subspace of $l_{\infty}(S)$ generated by the characteristic functions, then $\psi$ agrees with the $\mu_{E}{ }^{-}$ integral on $V$. The fact that $V$ is norm-dense in $l_{\infty}(S)$ implies that $\psi$ and the $\mu_{E}$-integral are equal on all of $l_{\infty}(S)$.

Now let $B$ be a $\sigma$-algebra of subsets of $S$ and let $\mu$ be a countably additive probability measure on $\mathbb{B}$ which satisfies $\mu(A)=0$ for each finite set $A$ in $B$. There exists an extension $\tilde{\mu}$ of $\mu$ to $\mathscr{P}(S)$ which satisfies $\tilde{\mu}(\{s\})=0$ for $s \in S$. By Theorem 1 , there exists a ${ }^{*}$-finite subset $F$ of ${ }^{*} S$ which satisfies $\tilde{\mu}=\mu_{F}$, and thus $\mu(A)=\mu_{F}(A)$ for every $A$ in $B$.

For any bounded, $\mu$-integrable function $f, \int f d \mu=\int f d \tilde{\mu}$. Therefore, by Lemma 1,

$$
\int f d \mu=\mathrm{st}\left(\frac{1}{\|F\|} \sum_{p \in F} *_{f}(p)\right) .
$$

However, for unbounded, $\mu$-integrable functions (6) may not be true. (Indeed, if $f$ is any unbounded function on $S$, then $F$ may be chosen satisfying $\mu=\mu_{F}$ on $B$, but such that the sum $\|F\|^{-1} \Sigma_{p \in F}{ }^{*} f(p)$ is infinite.) It is possible, nonetheless, to choose $F$ in such a way that (6) is true for every $\mu$-integrable function.

It is convenient to assume that $* M$ is $\kappa$-saturated (in the sense of [7]), where $\kappa$ is any cardinal number greater than the number of functions from $S$ to $R$. The remainder of this section is devoted to showing that, under this assumption, it is possible to represent $\mu$ on $\mathfrak{B}$ in such a way that (6) holds for every $\mu$-integrable function.

Given $n \in N$ and a function $f$ from $S$ to $R$, define $f_{n}$ on $S$ by

$$
f_{n}(x)= \begin{cases}f(x) & \text { if }|f(x)| \leq n \\ 0 & \text { otherwise }\end{cases}
$$


Each $f_{n}$ is a bounded function, and it is measurable whenever $f$ is. Also, if $\omega \epsilon^{*} N$ and $p \in{ }^{*} S$, then

$$
*_{\omega}(p)= \begin{cases}* f(p) & \text { if }|* f(p)| \leq \omega, \\ 0 & \text { otherwise. }\end{cases}
$$

Lemma 2. Let $E$ be any *finite subset of ${ }^{*} S$ which satisfies $\mu=\mu_{E}$ on $\Re$ and let $f$ be a nonnegative, $\mu$-integrable function. There exists an internal sub. set $F_{f}$ of $E$ which satisfies $\left\|F_{f}\right\| /\|E\|==_{1} 1$ and, for any internal subset $F$ of $F_{f}$,

$$
\frac{\|F\|}{\|E\|}={ }_{1} 1 \rightarrow \int f d \mu=\operatorname{st}\left(\frac{1}{\|F\|} \sum_{p \in F} * f(p)\right) \text {. }
$$

Proof. For each $n \in N$, let $A_{n}=\{x \mid f(x)>n\}$. Then $\left\{A_{n} \mid n \in N\right\}$ is a decreasing chain of sets in $\mathscr{B}$ and $\bigcap\left\{A_{n} \mid n \in N\right\}=\varnothing$. Thus the sequence $\left\{\mu\left(A_{n}\right)\right\}$ decreases monotonically to 0 . Since $\mu=\mu_{E}$ on $\Re$, it follows that for each $\delta>0$ in $R$, there exists $n_{0} \in N$ which satisfies

$$
n \geq n_{0} \rightarrow\left\|* A_{n} \cap E\right\| /\|E\|<\delta
$$

If $\omega$ is an infinite member of ${ }^{*} N$, then ${ }^{*} A_{\omega} \subset{ }^{*} A_{n}$, so $\left\|^{*} A_{\omega} \cap E\right\| /\|E\|<\delta$.

This shows that for every such $\omega$,

$$
\left\|^{*} A_{\omega} \cap E\right\| /\|E\|={ }_{1} 0 .
$$

Since $f$ is nonnegative, the sequence of integrals $\int f_{n} d \mu$ is increasing. By the monotone convergence theorem, the supremum of this sequence is $\int f d \mu$. If $\int f d \mu=\int f_{n} d \mu$ for some $n \in N$, then $\mu\left(A_{n}\right)=0$ and hence

$$
\left\|E \sim *_{A_{n}}\right\| /\|E\|={ }_{1} 1 \text {. }
$$

In this case let $F_{f}=E \sim{ }^{*} A_{n^{*}}$. If $F \subset F_{f}$ and $\|F\| /\|E\|={ }_{1} 1$, then

$$
\int f d \mu=\int f_{n} d \mu=s t\left(\frac{1}{\|F\|} \sum_{p \in F} * f(p)\right)
$$

since ${ }^{*} f={ }^{*} f_{n}$ on $F$ and $\mu_{F}=\mu_{E}$.

Therefore it may be assumed that $\int f_{n} d \mu<\int f d \mu$ for all $n \in N$. Thus

$$
\frac{1}{\|E\|} \sum_{p \in E} * f_{n}(p)<\int f d \mu
$$

for all $n \in N$. It follows that there is an infinite $\omega$ in ${ }^{*} N$ which satisfies

$$
\frac{1}{\|E\|} \sum_{p \in E} *_{\omega}(p)<\int f d \mu .
$$

In this case let $F_{f}=E \sim{ }^{*} A_{\omega}$, so that $\left\|F_{f}\right\| /\|E\|={ }_{1} 1$ by (7). Suppose $F$ is any internal subset of $F$, which satisfies $\|F\| /\|E\|={ }_{1} 1$. Then, for each $n \in N$, 


$$
\begin{aligned}
\int f_{n} d \mu & \leq \mathrm{st}\left(\frac{1}{\|F\|} \sum_{p \in F} *_{f}(p)\right) \\
& \leq \operatorname{st}\left(\frac{1}{\|E\|} \sum_{p \in E} *_{\omega}(p)\right)=\int f d \mu,
\end{aligned}
$$

using Lemma 1 and the fact that ${ }^{*} f={ }^{*} f_{\omega}$ on $F$. By the monotone convergence theorem

$$
\text { st }\left(\frac{1}{\|F\|} \sum_{p \in F} * f(p)\right)=\int f d \mu
$$

completing the proof.

Theorem 2. Let $B$ be an $\sigma$-algebra of subsets of $S$ and let $\mu$ be a countably additive probability measure on $\mathbb{B}$ which satisfies $\mu(A)=0$ for each finite set $A$ in $B$. There exists $a{ }^{*}$-finite subset $F$ of ${ }^{*} S$ which satisfies $\mu=\mu_{F}$ on $\mathfrak{B}$ and

$$
\int f d \mu=s t\left(\frac{1}{\|F\|} \sum_{p \in F} * f(p)\right)
$$

for every $\mu$-integrable function $f$.

Proof. Let $I$ be the set of nonnegative, $\mu$-integrable functions. Since each $\mu$-integrable function is the difference of two elements of $I$, it suffices to find an $F$ which satisfies the conditions of the theorem for every $f$ in $l$. By Theorem 1 (and the remarks following) there exists a ${ }^{*}$-finite subset $E$ of ${ }^{*} S$ which satisfies $\mu=\mu_{E}$ on $\mathfrak{B}$. For each $f \in I$, let $F$, be a subset of $E$ which satisfies the conditions of Lemma 2. Given $n \in N$ and $f \in I$, define

$$
A(n, f)=\left\{F \mid F \text { is an internal subset of } F_{f} \text { and }\|F\| /\|E\|>n /(n+1)\right\} .
$$

This family of internal sets has cardinality card $(N \times I)$, which is less than $\kappa$. Moreover, the family has the finite intersection property. $\left(F_{f_{1}} \cap \ldots \cap F_{f_{n}}\right.$ is an element of $A\left(m_{1}, f_{1}\right) \cap \ldots \cap A\left(m_{n}, f_{n}\right)$ whenever $m_{1}, \cdots, m_{n} \in N$ and $f_{1}, \cdots, f_{n} \in I$.) Since ${ }^{*} \mathbb{M}$ is $\kappa$-saturated, there exists a ${ }^{*}$-finite set $F$ which satisfies $F \in A(n, f)$ for every $n \in N$ and $f \in I$ (Theorem 2.7.12 of [5]). That is, $F \subset F_{f}$ for every $f \in I$, and $\|F\| /\|E\|={ }_{1} 1$. It follows by Lemma 2 that $F$ satisfies the conditions of the theorem.

Remark. Theorem 2 is true even if ${ }^{*} \pi$ is not $\kappa$-saturated, but the proof of that fact is somewhat more complicated. The proof given here proves the stronger result that $F$ can be chosen as a subset of any given set $E$ which satisfies $\mu=\mu_{E}$ on $B$. 
2. An application. The following standard result can be proved easily using the Riesz Representation Theorem. The nonstandard proof given here uses the extension to $l_{\infty}(S)$ of Robinson's representation result [9] instead.

Theorem 3. Let $X$ be a compact, Hausdorff space, $\left\{f_{n}\right\}$ a sequence in $C(X)$ and $\phi$ a bounded linear functional on $C(X)$. If $\left\{f_{n}\right\}$ is uniformly bounded on $X$ and converges to 0 pointwise, then $\phi\left(f_{n}\right) \rightarrow 0$.

Proof. Let $\phi$ be any bounded linear functional on $C(X)$. By the Hahn-Banach theorem, $\phi$ may be extended to a bounded linear functional $\tilde{\phi}$ on $l_{\infty}(X)$. By the extension to $l_{\infty}(X)$ of the principal result of [9], there exist a ${ }^{*}$-finite subset of ${ }^{*} X$ and an internal function $\lambda$ from $F$ into ${ }^{*} R$ which satisfy

$$
\tilde{\phi}(f)=s t\left(\sum_{p \in F} \lambda(p) * f(p)\right)
$$

for every $f$ in $l_{\infty}(X)$, and $\Sigma_{p \in F}|\lambda(p)|$ is finite.

Let $\left\{f_{n}\right\}$ be a sequence in $C(X)$ which is uniformly bounded on $X$ by 1 , and which converges to 0 , pointwise. If $\phi\left(f_{n}\right)$ does not converge to 0 , then it may be assumed (by taking a subsequence) that for some $\delta>0$ in $R,\left|\phi\left(f_{n}\right)\right|>\delta$ for every $n \in N$. Let $M=$ st $\left(\Sigma_{p \in F}|\lambda(p)|\right)+1$. For $n \in N$, define

$$
A_{n}=\left\{x \mid x \in X \text { and }\left|f_{n}(x)\right| \geq \delta / 2 M\right\} \text {. }
$$

Therefore,

$$
\begin{aligned}
\delta & <\left|\sum_{p \in F} \lambda(p) * f_{n}(p)\right| \\
& \leq \sum_{p \in * A_{n} \cap F}\left|\lambda(p) * f_{n}(p)\right|+\sum_{p \in F \sim * A_{n}}\left|\lambda(p) * f_{n}(p)\right| \\
& \leq \sum_{p \in * A_{n} \cap F}|\lambda(p)|+\frac{\delta}{2} .
\end{aligned}
$$

Thus, for each $n \in N, \Sigma_{p \epsilon^{*} A_{n} \cap F}|\lambda(p)|>\delta / 2$.

Now define $\mu^{\prime}$ on $\mathscr{P}(X)$ by

$$
\mu^{\prime}(A)=\operatorname{st}\left(\sum_{p \in *^{*} A \cap F}|\lambda(p)|\right)
$$

for each $A \subset X$. Then $\mu^{\prime}$ is a measure on $\mathcal{P}(X)$, and $\mu^{\prime}\left(A_{n}\right)>\delta / 2$ for every $n \in N$. It follows that there is an infinite subset $K$ of $N$ such that $\left\{A_{n} \mid n \in K\right\}$ has the finite intersection property (see Lemma 17.9 of [4]). Since $* M^{n}$ is an enlargement, there is an element $p$ of ${ }^{*} X$ which satisfies $\left|{ }^{*} f(p)\right| \geq \delta / 2 M$ for all $n \in K . X$ is compact, so $p$ is near-standard to some $x \in X$. In particular, ${ }^{*} f_{n}(p)={ }_{1} f_{n}(x)$ for every $n \in N$. This implies $\left|f_{n}(x)\right| \geq \delta / 2 M$ for every $n \in K$, 
which contradicts the assumption that $f_{n}(x)$ converges to 0 . Therefore $\phi\left(f_{n}\right)$ must converge to 0 .

3. Constructing invariant measures. Let $G$ be a group of permutations on $S$, and assume that $G$ satisfies Følner's condition:

For each $a_{1}, \cdots, a_{n} \in G$ and $k \in N$, there exists a finite set $A \subset G$ which satisfies $\left\|A \triangle A a{ }_{j}\right\| /\|A\|<1 /(k+1)$ for each $j=1, \ldots, n$.

To apply the corresponding statement in ${ }^{*} \pi$, let $E$ be a ${ }^{*}$-finite subset of ${ }^{*} G$ which contains $\left\{{ }^{*} g \mid g \in G\right\}$ and let $\omega$ be an infinite member of ${ }^{*} N$. Then there is a ${ }^{*}$-finite set $F C^{*} G$ which satisfies $\|F \triangle F p\| /\|F\|<1 / \omega$ for every $p \in E$. In particular,

$$
g \in G \rightarrow\left\|F \triangle F^{*} g\right\| /\|F\|={ }_{1} 0 .
$$

If $F$ satisfies (8), then $\mu_{F}$ is a probability measure on $\mathscr{P}(G)$ and $\mu_{F}$ is invariant under the action of $G$ on itself by right multiplication. The principal result of [3] is, essentially, that the converse holds: If there is such a measure on $\mathcal{P}(G)$, then $G$ satisfies $\mathrm{F} \phi$ lner's condition.

Theorem 4. Let $G$ be a group of permutations of $S$ and let $F$ be a *-finite subset of ${ }^{*} G$ which satisfies (8). Let $\mu$ be any measure on $\mathcal{P}(S)$ and define $\tilde{\mu}$ by

$$
\tilde{\mu}(A)=s t\left(\frac{1}{\|F\|} \sum_{p \in F} *_{\mu}\left(p^{*} A\right)\right)
$$

for $A \subset S$. Then $\tilde{\mu}$ is a $G$-invariant measure on $\mathcal{P}(S)$. Moreover, if $A \subset S$ satisfies $\mu(g A)=\mu(A)$ for every $g \in G$, then $\tilde{\mu}(A)=\mu(A)$.

Proof. Each element of ${ }^{*} G$ is a permutation of ${ }^{*} S$. Thus if $A, B$ are disjoint subsets of $S$, then $p^{*} A, p^{*} B$ are disjoint subsets of ${ }^{*} S$ for each $p \in{ }^{*} G$. Thus ${ }^{*} \mu\left(p\left({ }^{*} A \cup{ }^{*} B\right)\right)={ }^{*} \mu\left(p^{*} A\right)+{ }^{*} \mu\left(p^{*} B\right)$. From this the finite additivity of $\tilde{\mu}$ is immediate.

Given $A$ in $\mathcal{P}(S)$ and $g$ in $G$,

$$
\begin{aligned}
|\tilde{\mu}(g A)-\tilde{\mu}(A)| & =1\left|\frac{1}{\|F\|} \sum_{p \in F}\left(*^{*}\left(p^{*} g^{*} A\right)-*_{\mu}\left(p^{*} A\right)\right)\right| \\
& \leq \frac{1}{\|F\|} \sum_{p \in F \Delta F^{*} g} *_{\mu}\left(p^{*} A\right) \\
& \leq \mu(S) \cdot\left\|F \triangle F^{*} g\right\| /\|F\|={ }_{1} 0 .
\end{aligned}
$$

Therefore $\tilde{\mu}(g A)=\tilde{\mu}(A)$, so that $\tilde{\mu}$ is $G$-invariant.

Finally, suppose $A$ is a subset of $S$ which satisfies $\mu(g A)=\mu(A)$ for every $g \in G$. Then ${ }^{*} \mu\left(p^{*} A\right)={ }^{*} \mu\left({ }^{*} A\right)$ for every $p \in{ }^{*} G$. Therefore 


$$
\tilde{\mu}(A)=\operatorname{st}\left(\frac{1}{\|F\|} \sum_{p \in F} *_{\mu}(* A)\right)=\mu(A) .
$$

To prove Banach's extension result, let $G$ be the group of all translations $(\bmod 1)$ of $[0,1]$, and let $\mu$ be any extension of Lebesgue measure to $\mathscr{P}([0,1])$. It is well known, and easy to prove using the decomposition theorem for finitely generated abelian groups, that every abelian group satisfies F $\phi$ lner's condition. Since $G$ is abelian, Theorem 4 can be applied to obtain a $G$-invariant measure $\tilde{\mu}$ on $\mathscr{P}([0,1])$. If $A$ is a Lebesgue measurable subset of $[0,1]$, then $\mu(g A)=$ $\mu(A)$ for every $g \in G$. Theorem 4 thus asserts that $\tilde{\mu}(A)=\mu(A)$; that is, $\tilde{\mu}$ is an extension of Lebesgue measure.

Acknowledgement. The author is indebted to L. C. Moore for some helpful conversations on the subject of this paper, and for a suggestion which simplified the proof of Theorem 3 .

\section{REFERENCES}

1. S. Banach, Sur le problème c, la mesure, Fund. Math. 4 (1923), 7-33.

2. A. R. Bernstein and F. Wattenberg, Nonstandard measure theory, Internat. Sympos. Applications of Model Theory to Algebra, Analysis, and Probability (Pasadena, Calif., 1967), Holt, Rinehart and Winston, New York, 1969, pp. 171-185. MR 40 \#287.

3. E. Følner, On groups with full Banach mean value, Math. Scand. 3 (1955), 243-254. MR 18, 51 .

4. J. L. Kelley and I. Namioka, Linear topological spaces, University Series in Higher Math., Van Nostrand, Princeton, N. J., 1963. MR 29 \#3851.

5. P. A. Loeb, A nonstandard representation of measurable spaces and $L_{\infty}$, Bull. Amer. Math. Soc. 77 (1971), 540-544. MR 43 \#2488.

6. - A nonstandard representation of measurable spaces, $L_{\infty}$ and $L_{\infty}^{*}$, Contributions to Nonstandard Analysis, North-Holland, Amsterdam, 1972.

7. W. A. J. Luxemburg, A general theory of monads, Internat. Sympos. Applications of Model Theory to Algebra, Analysis, and Probability (Pasadena, Calif., 1967), Holt, Rinehart and Winston, New York, 1969, pp. 18-86. MR 39 \#6244.

8. M. Machover and J. Hirschfeld, Lectures on nonstandard analysis, Lecture Notes in Math., vol. 94, Springer-Verlag, Berlin and New York, 1969. MR $40 \# 2531$.

9. A. Robinson, On generalized limits and linear functionals, Pacific J. Math. 14 (1964), 269-283. MR $29 \# 1534$.

10. - Non-standard analysis, North-Holland, Amsterdam, 1966. MR $34 \# 5680$.

DEPARTMENT OF MATHEMATICS, DUKE UNIVERSITY, DURHAM, NORTH CAROLINA 